\title{
Editorial to first issue
}

\author{
Vincenzo Loia
}

Published online: 27 January 2010

(C) Springer-Verlag 2010

On behalf of the Editorial Board, it is with great pride and sincere privilege that I am writing this message to present the first issue of the Journal of Ambient Intelligence and Humanized Computing. Launching this new journal would not have been possible without the great and much appreciated contributions from the Editorial Board members and from Springer. Ambient Intelligence (AmI) has acquired great importance in recent years and requires the development of new innovative approaches to the challenges of the twenty-first century. Many leading advisory IT companies and a wide researcher community predict a deep acceleration of AmI wave that will lead to emergent scenarios as significant as the Web. The journal aims to provide a common platform for researchers to promptly share their novel results and latest developments in Ambient Intelligence and Humanized Computing, built on advances in engineering, computer sciences, robotics, mechatronics, social sciences, cognitive sciences, physics, arts, architecture and economy. The journal wishes to serve as an international forum for all issues within the editorial focus, in an effort to disseminate the results guaranteeing scientific excellence. Currently the Journal is open to the following scientific contributions:

- Pervasive/Ubiquitous Computing and Applications

- Cognitive wireless sensor network

- Embedded Systems and Software

- Mobile Computing and Wireless Communications

- Next Generation Multimedia Systems

- Security, Privacy and Trust

- Service and Semantic Computing

V. Loia $(\square)$

University of Salerno, Salerno, Italy

e-mail: loia@unisa.it
- Advanced Networking Architectures

- Dependable, Reliable and Autonomic Computing

- Embedded Smart Agents

- Context awareness, social sensing and inference

- Multi modal interaction design

- Ergonomics and product prototyping

- Intelligent and self-organizing transportation networks and services

- Healthcare Systems

- Virtual Humans and Virtual Worlds.

This inaugural issue features six scientific papers. The first contribution, by T. K. R. Nkwe and M. K. Denko, addresses the problem of gateway bottleneck by using data management techniques. The authors propose a selfoptimizing cooperative caching solution for autonomic wireless mesh networks (WMNs) capable to improve the data caching performance providing data ubiquity.

The second paper, by M. J. O'Grady, C. Muldoon, M. Dragone, R. Tynan, and G. M. P. O'Hare, explores Ambient Assisted Living (AAL) from a wide number of prospectives. Developments in AAL over the next few years will have a profound affect on society and the elderly in particular. Decisions made at this time juncture in the design of AAL systems may well have ramifications for years to come, considering appropriate solutions capable to support flexibility, scalability and evolution during the aging process. In particular, the authors discuss an agentbased middleware approach to realising an architecture for evolutionary AAL.

The third paper, by T. Gross, discusses on Cooperative ambient intelligence. Cooperative ambient intelligence aims to improve users' work and private life by analysing their current situation with a special focus on their social interaction and to adapt the environment accordingly. 
As technology is seen as highly embedded in physical environments and the social fabric of users, a reconsideration of methods for its design, implementation, and evaluation is vital. The author clarifies his view on cooperative ambient intelligence, and discuss what constitutes a novel human-centred computing methodology.

The fourth paper, by M. Kaptein, P. Markopoulos, B. de Ruyter, and E. Aarts, discusses ambient persuasion and poses a model that structures the knowledge from social sciences on persuasion, attitude change, and behavior change. This article provides an overview of the social science findings on attitude and behavioral change and summarize these in a model for ambient persuasion. The authors deepen the discussion on each part of the model, identifying major challenges for the AmI field to fulfill its opportunities in incorporating persuasion.

The fifth paper, by G. Singla, D. J. Cook, and M. Schmitter-Edgecombe, describes an approach to recognizing activities performed by smart home residents. In particular, the authors present in detail novel algorithms that built probabilistic models of activities and used them to recognize activities in complex situations where multiple residents are performing activities in parallel in the same environment.

Finally, the paper of W. Pedrycz, investigates the capabilities of Granular Computing (and fuzzy sets, in particular) that are available in the currently existing framework to support the design of human-centric systems. Information granules constitute a backbone or a conceptual skeleton of a variety of ways we perceive the world, communicate our findings and revise/update and organize our knowledge. This work proposes an enhanced platform of human-centric processing, detailing how to capture and represent information knowledge in terms of higher type constructs and their use in complex decision-making activities.

The birth of a new journal comes from a long process and we took all the necessary steps to make it a highcalibre scientific publication. We are relying on the collaboration of all our Editors, reviewers and contributors to make it a contemporary, lively and relevant publication.

We hope you will enjoy reading our first issue, and that you find these articles useful to stimulate your research into the vibrant area of Ambient Intelligence and Humanized Computing. We invite you to submit your best papers for publication.

With my kindest regards,

Vincenzo Loia

Editor-in-Chief

University of Salerno, Italy 